\title{
Conference Summary: HI Science in the Next Decade
}

\author{
Martha P. Haynes \\ 530 Space Sciences Building, Cornell University, Ithaca NY 14853
}

\begin{abstract}
The atomic hydrogen (HI) $21 \mathrm{~cm}$ line measures the gas content within and around galaxies, traces the dark matter potential and probes volumes and objects that other surveys do not. Over the next decade, $21 \mathrm{~cm}$ line science will exploit new technologies, especially focal plane and aperture arrays, and will see the deployment of Epoch of Reionization/Dark Age detection experiments and Square Kilometer Array (SKA) precursor instruments. Several experiments designed to detect and eventually to characterize the reionization history of the intergalactic medium should deliver first results within two-three years time. Although "precision cosmology" surveys of $\mathrm{HI}$ in galaxies at $z \sim 1$ to 3 require the full collecting area of the SKA, a coherent program of HI line science making use of the unique capabilities of both the existing facilities and the novel ones demonstrated by the SKA precursors will teach us how many gas rich galaxies there really are and where they reside and will yield fundamental insight into how galaxies accrete gas, form stars and interact with their environment.
\end{abstract}

Keywords: HI line, Reionization, Gas Content, Galaxy Surveys, HI Mass Function

PACS: 98.38.Gt,98.62.Ai,98.62.Ve,98.80.Es

\section{INTRODUCTION}

Huge surveys being undertaken at optical and near-IR wavelengths today are cataloguing and characterizing many millions of galaxies, feeding their colors and structural properties into sophisticated models that describe their evolution over cosmic time. In contrast to the wealth of detail available for stellar components of galaxies, quantitative measures of the gas content are limited to only a few tens of thousands of objects, and of the gas distribution within them, to only hundreds. Yet, galaxies and the stars within them form out of collapsing gas clouds and both the amount of gas and its distribution within and around galaxies play critical roles in the story of galaxy evolution.

But, how important really is the HI $21 \mathrm{~cm}$ line? Sometimes at topical meetings such as this one, the attendees are mainly "the usual suspects" and the elder participants spend most of their time remembering the "good old days". Perhaps to unsettle any tendency towards scientific smugness about the long tradition of $21 \mathrm{~cm}$ line studies, Riccardo Giovanelli, right at the beginning of this conference, reminded us that HI contributes a "piffling" amount even to the overall baryon budget in the universe. If that were not enough to deflate our HI egos, Todd Tripp rightly pointed out that Ly $\alpha$ absorption lines are a factor of $10^{6}$ times more sensitive to low column density gas than are typical HI maps, and numerous speakers reminded us that nearly every map we make in HI pales in spatial detail to the minutely elegant structures gleaned from the pretty images made with "real" telescopes. And, as if to render the conference pointless, Leo Blitz questioned whether $\mathrm{HI}$ is really relevant to studies of star formation, since stars form out of $\mathrm{H}_{2}$, not 
HI. With this self-skepticism as a backdrop to this conference, speakers and participants engaged in healthy discussion of the relative importance of $\mathrm{HI}$ and other techniques to cosmology and galaxy studies. The often-lively discussion was not just of what we do and don't know, but most importantly, what we might know in the next 5-10 years. It was that focus on peering into the HI crystal ball that was most appealing: where is HI science headed and how can we expedite the voyage? In this review, I focus on the outcome of those discussions, especially as they relate to the the US-based facilities.

\section{THE 21ST CENTURY: A NEW AGE IN HI SPECTROSCOPY}

Two advantages of the radio $21 \mathrm{~cm}$ line are its simple physics and potential detectability over a wide redshift range by a single (huge) telescope operating in the $\mathrm{cm}-\mathrm{m} \lambda$ wavelength range. So, if HI is so "simple", why is its application as a tracer of cosmic evolution so seemingly far behind OIR studies in terms of redshift numbers and depth? The prime reason is the lack of facilities: without significant collecting area, HI emission is hard to detect at large distances or low column densities. In contrast to the science potential of small optical telescopes, single small radio telescopes cannot contribute to HI studies much beyond the Milky Way. For nearly all purposes, HI "telescopes" must be big (in terms of collecting area), and hence, are expensive both to build and to operate. In practice, instruments rarely achieve optimal performance in all of the relevant parameters: sensitivity, sky coverage, angular resolution, field of view, spectral bandwidth and resolution. Practical trade-offs compromise some aspect of the science, e.g., collecting area versus angular resolution, total bandwidth versus spectral resolution. And, while radio waves are not affected by terrestrial cloud cover, we live on a planet whose inhabitants create an increasingly noisy radio environment and in a galaxy which emits a strong synchrotron foreground.

Most, if not all, of the participants of this conference agree that the objective for construction of the next major ground-based facility of direct relevance to HI science is the "Square Kilometer Array" (SKA). With something like a square kilometer of collecting area, an HI line survey of a billion galaxies from $z=0$ to 2 or more, as discussed at this meeting by Joseph Lazio, Steve Myers and Rogier Windhorst, will be possible. Such a survey, with a peak redshift distribution at $z \sim 1.5$, will someday allow "precision cosmology" measures of the baryonic acoustic oscillations by an entirely independent spectroscopic technique as discussed by the Dark Energy Task Force [1], while at the same time tracing the evolution of the galaxy population at epochs where their baryon content was gas-dominated. Who wouldn't want to be part of that great adventure?

The 21 st century is bringing to $\mathrm{cm}-\mathrm{m} \lambda$ astronomy new opportunities driven principally by advances in computational resources and the development of array technologies. The latter fall into two quite separate categories: (1) arrays of beams which occupy the focal plane: feed arrays in which individual feed horns contribute pixels and focal plane phased arrays which manipulate the electric field digitally to "form" beams; and (2) aperture arrays, which use separate antenna elements to synthesize collecting area. Some of the planned future instruments will incorporate both kinds of array technologies to maximum advantage. Attach these devices to digital correlators, add advanced 
computational capability, adopt clever observing strategies and develop powerful signal processing algorithms, and you get the SKA, the only trick being that right now, we are still a bit ahead of the technology wave required to get us our billion galaxies. Achieving a square kilometer of collecting area over the entire $\mathrm{cm}-\mathrm{m} \lambda$ range with a single facility is an extremely expensive and probably unrealistic proposition. Rather than discussing what we meant by "the" SKA, we talked about steps to enable transformational "SKA-class" $21 \mathrm{~cm} \lambda$ HI science, both through the development of "SKA precursors" and through the exploitation of new technologies on the existing large collecting areas, particularly the Arecibo $305 \mathrm{~m}$ antenna and the EVLA (Expanded Very Large Array).

Technologically ambitious new instruments including ASKAP (the Australian Square Kilometre Array Pathfinder), ATA-350 (the Allen Telescope Array expansion), FAST (Five-Hundred-meter Aperture Spherical Telescope), MeerKat (the Karoo Array Telescope), LOFAR (Low Frequency Array), and MWA (Murchison Widefield Array), all currently under construction, promise to validate new technologies and to demonstrate new science capabilities across the $\mathrm{cm}-\mathrm{m} \lambda$ range. Each of these facilities will bring new strengths geared toward particular applications ranging from low frequency operations to ultrabroad frequency coverage to wide field imaging. The potential scientific achievement of this suite of new instruments is quite astounding. Because they are truly revolutionary, much technical work remains to be done before their full capabilities can be realized. In equally impressive ways, innovative advances in frontends, backends and signal processing capabilities are making possible gigantic improvements in our ability to explore the extragalactic HI universe using the current world-class facilities. Where the EVLA and Arecibo demonstrate SKA technologies and techniques to explore SKAclass science, they too should be considered SKA precursors and we should exploit them as such.

The Arecibo $305 \mathrm{~m}$ dish remains the world's largest collecting area at $\mathrm{cm}-\mathrm{m} \lambda$. As a single aperture, its strength lies in the detection of weak sources, exploiting its gain advantage. The advent of the Arecibo L-band Feed Array (ALFA) has thoroughly revolutionized not only the kind of surveys underway at Arecibo but also how they are being done. John Stocke remarked on my career-long predisposition to drift scan observing; like the Sloan Digital Sky Survey, the Arecibo Legacy Fast ALFA (ALFALFA) survey[2], demonstrates that "keeping it simple" delivers superior data quality in a highly efficient manner. An innovation of ALFALFA is the full integration of public multiwavelength databases using Virtual Observatory tools into the analysis process, allowing its catalogs to include both the HI and optical identifications, where such exist. The comparison of HIPASS (Parkes HI All-Sky Survey[3] and ALFALFA[4] [5], discussed by Riccardo Giovanelli, emphasizes the impact of multibeaming on Arecibo's huge collecting area. The deployment of focal plane phased arrays, when that technology becomes available, will energize a similar revolution. The development of these devices is vital not only to Arecibo but also to several of the SKA precursor projects, notably ASKAP, FAST and the APERTIF (Aperture Tile in Focus) upgrade to the Westerbork telescope discussed by Thijs van der Hulst.

Similarly, HI programs are poised to exploit the EVLA in truly exciting ways. Until now, the VLA correlator could not simultaneously deliver adequate bandwidth even to cover the entire velocity range of a typical galaxy cluster and velocity resolution was frequently compromised to a point biased against the lowest mass, narrowest linewidth 
systems. The new EVLA correlator will offer a huge advance for, among other things, HI studies of galaxies in and near groups and clusters and deep searches for HI in emission or absorption, with the potential for commensal observing with all L-band EVLA programs over the full range from $z=0$ to 0.5 . Its greatly increased capabiliities for high dynamic range imaging will permit study of $\mathrm{HI}$ in the most active, radio loud systems and searches for very weak absorption lines. An innovative technical advance to provide prime focus capability would allow the EVLA to explore the earlier times $z>1$, when unlike today, most of the baryons in galaxies were in the gaseous component.

As already demonstrated by the on-going ALFA surveys, commensal observing offers a tremendous increase in the efficiency potential of $\mathrm{cm} \lambda$ instruments. The voltage detected by a radio telescope can be mixed, amplified and sent independently to multiple digital backends without loss of signal, so that the same patch of sky can be examined for its continuum, time-variable and spectroscopic signatures simultaneously. As demonstrated by the commensal ALFA surveys being undertaken today at Arecibo, we should expand the design of programs which can exploit this unique feature of radio astronomy. Currently, the galactic HI TOGS program runs commensally with ALFALFA; by the end of this year, the PALFA pulsar survey will turn on a third spectrometer. What a remarkable use of a mode that already delivers $99 \%$ open shutter time!

The SKA is where we are headed, but the path from here to there must include science productivity along the way. It would be silly not to work hard toward SKA development; that billion galaxy survey will be that revolutionary. But, I suggest that a square kilometer of collecting area is such a huge leap that we do not yet understand the evolution of galaxies adequately to know how to exploit it fully. So, let's use what we already have to head down the SKA science path. In the next section, I discuss some considerations and propose some specific approaches which clearly lead toward the SKA but keep up a sustained pace of scientific discovery along the way.

\section{HI SURVEYS: TRACING THE EVOLUTION OF THE GAS}

The new $\mathrm{cm}-\mathrm{m} \lambda$ instruments currently under construction are specifically designed to achieve, among other things, the characteristics required for the undertaking of major $\mathrm{HI}$ surveys. Like the SKA, most of them are intended to be used in commensal mode, particularly with time domain surveys as described in the RSSKA (Radio Synoptic Square Kilometer Array) concept presented at the conference by Steve Myers. We can assume that they will deliver superb science, although exactly what surveys they will undertake probably remains to be decided based on final performance specifications and the compromises required to accommodate commensal survey operations. In addition to these entirely new instruments, the existing facilities are also being equipped with new capabilities which can be tapped to explore a set of different questions. I advocate a program of complementary surveys which, in sum, will explore the complex interrelationships of galaxies and their environments, stars and gas both within and outside galaxies. As a community, we should develop a strategy to exploit the few available facilities in an integrated, coherent science program to address the most fundamental questions. The SKA will provide its billion galaxy HI survey, but in the meantime, great progress can be made towards understanding the evolution of the gaseous constituent of galaxies through co- 
ordinated surveys of HI in carefully selected samples, targeting both individual objects, active, interacting and quiescent systems, galaxies in and near groups, clusters and voids and in deep fields. Here are four fundamental questions whose answers will elude us unless we view the universe through the HI window:

How and when did reionization occur? Assuming we understand anything about astrophysics, before there were stars in the universe, there was HI, and the most recent constraints set by WMAP5 [6] imply that reionization took place over an extended period of half a billion years. The potential for detection of HI emission from the Dark Ages and throughout the reionization era shares some analogy with Cosmic Microwave Background science in the early 1960's, just before the discovering of the 3K radiation. In the latter case, however, Penzias and Wilson were not deliberately seeking the CMB whereas, in the EOR case, the theorists are far ahead of the technique. The current "first generation" low frequency experiments designed specifically for this purpose hold great promise for detection, although we shouldn't underestimate the technical challenges. In the short term, multiple independent approaches offer the best strategy, until the first results are in, hopefully with multiple robust - and consistent - detections. The final design of a major EOR facility, beyond these experiments, must await their prior results, but it is probably safe to say that throughout the next decade, we should continue to add collecting area to the first set of instruments, depending on which ones pan out, what frequencies (redshifts) prove to be of most interest and what technical solutions offer the most promise.

How many gas-rich galaxies are there and where do they reside? Is there a primordial HI mass function (HIMF)? Are there gas-rich "missing satellites"? Do galaxies form in voids? One of the early results of ALFALFA is that its HI detection rate greatly exceeds that of pre-survey predictions based on HIMFs derived from other surveys. We still do not have adequate volume sampled to conclude whether this is a purely cosmological result or the product of superior data quality and signal processing software. However, past widely divergent and sometimes contradictory inferences on the slope of the low mass slope of the HIMF and its possible variation with extragalactic environment are symptoms of inadequate volume sampling. ALFALFA will provide cosmologically significant results on the HIMF overall and should detect several hundred objects with $\mathrm{M}_{H I}<10^{7.5} \mathrm{M}_{\odot}$. However, it is not sensitive enough to detect objects like Leo $\mathrm{T}$ at a distance greater than $2.5 \mathrm{Mpc}$. Remember that the first " $F$ " in ALFALFA stands for "fast" (its integration time is only $\sim 40 \mathrm{sec}$ per beam) and that, at the nearer distances, solid angle coverage is the best strategy to maximize the surveyed volume [2]. As suggested by Riccardo Giovanelli at this meeting, deeper, wide area Arecibo surveys should detect significant numbers of low HI mass galaxies throughout the Local Supercluster, especially in and near the Virgo cluster, and in the nearest voids. Among other things, Arecibo is fortuitously positioned so that Virgo is overhead, allowing a deep look at the central regions of the Local Supercluster, in and around the Virgo cluster. Synthesis instruments such as ASKAP, MeerKat, ATA and APERTIF will provide wide area surveys with the angular resolution needed to avoid confusion and to yield HI distributions. With a systematic, strategic approach, we could collaboratively survey the whole sky. I hope we will.

How and why do some galaxies accrete gas while others don't (anymore)? From other wavelength tracers, we can infer the star formation rate (SFR); star formation 
requires gas. So where did this gas come from and how did it reach a state of collapse? When and why did gas accretion stop? Which baryons end up in disks and which get blown away? Do interactions drive the evolution in the SFR density? What happens to the gas in major mergers? What processes dominate evolution in different volumes? Nearly all work to date on what is happening to galaxies at $z>1$ lacks quantitative measures of their gas content; our goal should be how the gas content of galaxies changes during the same epoch $z \sim 1$ to 0 that the SFR density appears to decline steeply. ALMA will contribute hugely to our understanding of the dense gas in galaxies and hence the star formation process, but ALMA is not a survey instrument and molecules need stars to "light them up". What about the gas not actively engaged (yet) in the star formation process? As we know from 30 years of studying HI deficiency, HI is an excellent tracer of the future potential for star formation. We need surveys which can provide measures of the HI mass as input to models of galaxy evolution to complement the constraints on stellar mass and star formation history contributed by colors and equivalent widths from O/IR/UV surveys. We also need a large, coordinated CO survey so that we can explore possible evolution of the molecular-to-atomic gas ratio.

The galaxies first detected in HI at high redshift will be massive systems. ALFALFA finds that $99 \%$ of high HI mass galaxies $\mathrm{M}_{H I}>10^{9.5}$ can be identified with an optical counterpart; indeed, there is no theoretical expectation that high mass halos which contain gas will not form stars. Hence, while HI blind surveys are extremely valuable for finding low mass, low surface brightness or low luminosity galaxies which are underrepresented in optically-selected samples, we can begin the study of the gas content of galaxies at higher redshift using targeted surveys selected by (known) optical criteria. Barbara Catinella has shown the viability of detecting HI emission in field galaxies at $z \sim 0.2-0.3$ with modest integration times using Arecibo's L-band wide receiver. The GALEX-Arecibo-SDSS Survey (GASS) discussed by David Schiminovich serves as a bridge between ALFALFA and higher redshift surveys. These Arecibo ventures are just a beginning; it is only a matter of telescope time (and, of course, telescope availability). We do need to be careful in designing surveys to make sure we do not focus purely on the most gas-rich populations. GASS, for example will explore galaxies to a limiting mass fraction, $\mathrm{M}_{H I} / \mathrm{M}_{*}$, so that we can begin to understand why some massive systems have red colors but still significant gas reservoirs. And, we should remember that not all early type galaxies are gas-poor: 40\% of the Local Supercluster E/SO's outside Virgo have HI detected by ALFALFA, if not coincident with their stellar components, then near them. Let's leave our minds open to surprises as to where we find HI.

We should exploit the synthesis instruments, most notably the EVLA, to study the distribution of $\mathrm{HI}$ in galaxies to probe a a wide range of environments especially in and around groups and clusters, in AGN-hosts and interacting systems. We need to understand how central supermassive black holes are fed by accreting gas and why there are relationships between global gas content and nuclear activity. We should also exploit the broad bandwidths of modern receiver systems to explore the highest redshifts via HI absorption line studies. First detection and then followup synthesis imaging can allow exploration of the physical conditions and kinematics within the host galaxies and in fortuitous circumstances of line-of-sight absorbers. Whatever surveys make sensible use of commensal programs should be undertaken; in those cases, our principal challenge may be to muster the bodies to process and analyze the acquired data. 
How do galaxies convert gas into stars? What regulates star formation in galaxies of different mass, luminosity and surface brightness and in different environments? How many stars result from feedback processes? Why does the HI gas track so well the dark matter? While HI reservoirs are typically found at large radii, star formation occurs at smaller radii and scale height where the molecular clouds reside. Yet, as Evan Skillman pointed out, although the HI column density has little to do with star formation in high mass galaxies, the opposite is true in dwarfs. In low gas column density disks, we need to understand what fraction of stars is formed as a result of local processes and what of global ones, why some dwarfs burst while others don't and how stars form in the faintest systems. Suggesting that the real question is "How did the gas arrive at its present state?", Evan challenged theories of star formation to explain both the presence and absence of gas. Have all gas-rich ellipticals acquired their gas only recently? Are the Local Group "transition dwarfs" on their way to becoming dSphs?

In combination with observations across the electromagnetic spectrum of the varied constituents associated with the star formation process, projects like SINGS, THINGS, LittleTHINGS, ANGST, etc are enabling detailed in situ tests of star formation laws. Coordination of these and future surveys so that they achieve the same spectral resolution, $\mathrm{u}-\mathrm{v}$ coverage, sensitivity etc promises the steady accumulation of an invaluable homogeneous dataset for comparative analysis of the HI distribution, kinematics and dynamics. Evan Skillman asked the astute question "How many such 'THINGS' do we need?" Certainly, many more than contained in the current HI synthesis collection. As Eric Wilcots has discussed, we need a mix of complete samples and of classes representative of dwarfs, early/late types, group/cluster galaxies, AGNs/starbursts, interacting systems and isolated objects, a full encyclopedia of the extragalactic HI zoo.

\section{SUMMARY OF THE SUMMARY}

At this workshop, we discussed what are the topics in extragalactic science for which $\mathrm{HI}$ is relevant and powerful and what real progress could we make in the near future. We agreed that the long term objective was more collecting area, but we also discussed how real transformational progress could be made within the next decade. HI studies are vital to the understanding of galaxy evolution and provide quantitative clues that no other wavelength can. To maximize the potential scientific payoff, we should, as a community, undertake $a$ set of coordinated large surveys that are fully integrated with efforts to study the stellar and gaseous components of galaxies via other techniques; some astronomical socialism will produce datasets which can be analyzed democratically. I do not think I am misrepresenting the general sentiment of the assembled group in claiming that a strategy for HI science over the next 5-10 years has three key elements: (1) undertaking of the best science with all of the SKA "precursors" to nail down the role of HI (and dark matter!) in the local universe and to begin exploration of the evolution of that role over cosmic time; (2) development of innovative technologies deployed on the existing and precursor facilities which will lead us toward the SKA; (3) engaging the broadest community to understand and challenge our understanding of how gas fuels star formation and galaxy evolution. The third element is the one least often discussed. Since the HI facilities we can use are few in number and shared with many other users, 
the undertaking of large survey programs must meet "legacy" standards but, for the health of our science, they must involve a broad cross-section of the community. Unless we are ready to turn over survey programs to a few individuals, we must figure out how to engage large groups in their undertaking without sacrificing quality within fiscal and practical boundaries. Our challenge cannot just be "what to do", it also must include "how to do it". And "how" demands "strategy", "balance" and "inclusiveness".

Neutral hydrogen may make up a "piffling" amount of the total matter in the universe, but its physical simplicity and the information it conveys about the gas content, distribution, kinematics and dynamics opens a powerful window on the evolution of galaxies, of the hierarchical structures in which they reside and of the universe itself.

\section{ACKNOWLEDGMENTS}

My participation in ALFALFA is supported by NSF grant AST-0607007 and by the Brinson Foundation. The Arecibo Observatory is part of the National Astronomy and Ionosphere Center which is operated by Cornell University under a cooperative agreement with the National Science Foundation. I thank the staff of the Arecibo Observatory for their ever warm and generous attention in support of this conference and especially Robert Minchin for inspiring and organizing it.

\section{REFERENCES}

1. Albrecht, A., et al., "Report of the Dark Energy Task Force", astro-ph/0609591.

2. Giovanelli, R. et al. 2005, AJ 130, 2598.

3. Barnes, D.G, et al., 2001, MNRAS, 322, 486.

4. Giovanelli, R. et al. 2007, AJ 133, 2569.

5. Saintonge, A. et al. 2008, AJ 135, 588.

6. Dunkley, J. et al. 2008, ApJS (submitted); astro-ph/0803.0586. 